\title{
String junction as a baryonic constituent
}

\author{
Yu.S.Kalashnikova, A.V.Nefediev ${ }^{\dagger}$ \\ Institute of Theoretical and Experimental Physics \\ 117259, Moscow,Russia
}

\begin{abstract}
We extend the model for QCD string with quarks to consider the Mercedes Benz string configuration describing the three-quark baryon. Under the assumption of adiabatic separation of quark and string junction motion we formulate and solve the classical equation of motion for the junction. We dare to quantize the motion of the junction, and discuss the impact of these modes on the baryon spectra.
\end{abstract}

The $q q q$ baryon, together with the $q \bar{q}$ meson, is usually considered in the framework of the constituent model as a simplest system with zero triality. It is clear, nevertheless, that in the QCD -motivated approach the only way to form gauge-invariant states with three quarks in the fundamental colour representation is to introduce the so-called string junction as an object that couples three quarks to make a colour singlet:

$$
\begin{aligned}
& \Psi\left(x_{1} x_{2} x_{3}, x_{0}\right)= \\
= & \psi^{\alpha}\left(x_{1}\right) \psi^{\beta}\left(x_{2}\right) \psi^{\gamma}\left(x_{3}\right) \Phi_{\alpha}^{\alpha^{\prime}}\left(x_{0}, x_{1}\right) \Phi_{\beta}^{\beta^{\prime}}\left(x_{0}, x_{2}\right) \Phi_{\gamma}^{\gamma^{\prime}}\left(x_{0}, x_{3}\right) \varepsilon_{\alpha^{\prime} \beta^{\prime} \gamma^{\prime}},
\end{aligned}
$$

where parallel transporters $\Phi$,

$$
\Phi_{\beta}^{\alpha}(x, y)=\left(P \exp i g \int_{y}^{x} B_{\mu}^{a} t^{a} d z_{\mu}\right)_{\beta}^{\alpha},
$$

stand to assure non-local gauge invariance of the $q q q$ system placed into non-trivial QCD background $B_{\mu}^{a}$. From this point of view it is obvious that the baryon should be treated as a simplest multiquark system containing, in comparison to the $q \bar{q}$ system, a new object with it's own dynamics. Standard way to deal with the junction dynamics is to assume that it always moves in such a way that the sum of distances between quarks and junction is minimal $[1,2]$. This assumption is motivated by non-relativistic approach, where confinement manifests itself as linear potential between constituents. On the other hand, the idea that a junction might be responsible for certain kinds of baryonic excitations was already discussed in [2].

*e-mail:yulia@vxitep.itep.ru

†e-mail:nefediev@vxitep.itep.ru 
In the present letter we relax the above-mentioned assumption on a junction following submissively the quarks. Actually our baryon is going to contain four constituents rather than three. Our starting point is the Vacuum Background Correlators method [3]. In the framework of this method the hadronic Green functions can be constructed starting from the QCD Lagrangian. The dynamics of interaction is defined by the averages of Wilson loop operators, and it may be shown that the area law asymptotic for Wilson loop average gives rise to the string-type interaction of the constituents. The model for the resulting straight-line $q \bar{q}$ string was considered in [4], and the hybrid mesonic excitations were analysed in [5].

To formulate the Hamiltonian approach to the problem one is to consider the Green function of a baryon,

$$
G\left(x_{1} x_{2} x_{3} x_{0}, y_{1} y_{2} y_{3} y_{0}\right)=<\Psi\left(x_{1} x_{2} x_{3}, x_{0}\right) \mid \Psi\left(y_{1} y_{2} y_{3}, y_{0}\right)>_{B}
$$

where the brackets mean the averaging over background field configurations. To define the effective action the Feynman-Schwinger representation [6] of a Green function (3) is used (in the Euclidean space):

$$
\begin{aligned}
G\left(x_{1} x_{2} x_{3} x_{0}, y_{1} y_{2} y_{3} y_{0}\right) & =\int_{0}^{\infty} d s_{1} \int_{0}^{\infty} d s_{2} \int_{0}^{\infty} d s_{3} \int D z_{1} D z_{2} D z_{3} \times \\
& \times \exp (-\mathcal{K}) \cdot<W>_{B}
\end{aligned}
$$

where

$$
\begin{gathered}
W=\varepsilon_{\alpha \beta \gamma} \Phi_{\alpha^{\prime}}^{\alpha}\left(\Gamma_{1}\right) \Phi_{\beta^{\prime}}^{\beta}\left(\Gamma_{2}\right) \Phi_{\gamma^{\prime}}^{\gamma}\left(\Gamma_{3}\right) \varepsilon^{\alpha^{\prime} \beta^{\prime} \gamma^{\prime}} \\
\mathcal{K}=\sum_{i=1}^{3}\left(m_{q_{i}}^{2} s_{i}+\frac{1}{4} \int_{0}^{s_{i}} \dot{z}_{i}^{2}(\tau) d \tau\right)
\end{gathered}
$$

boundary conditions are $z_{i}(0)=y_{i}, z_{i}\left(s_{i}\right)=x_{i}$, and the contours $\Gamma_{i}$ in the Wilson loop operator run over the trajectories of the quarks. Using the relation

$$
1=\frac{1}{3 !} \varepsilon_{\alpha_{1} \alpha_{2} \alpha_{3}} \varepsilon^{\beta_{1} \beta_{2} \beta_{3}} \Phi_{\beta_{1}}^{\alpha_{1}}(\Gamma) \Phi_{\beta_{2}}^{\alpha_{2}}(\Gamma) \Phi_{\beta_{3}}^{\alpha_{3}}(\Gamma)
$$

(which is due to the unimodularity condition for the $\mathrm{SU}(3)$ ), where $\Gamma$ is an arbitrary open path (the same for all three $\Phi$ 's) connecting points $x_{0}$ and $y_{0}$, we represent the Wilson loop in (4) as

$$
\begin{gathered}
W=S p W_{1} S p W_{2} S p W_{3}-S p\left(W_{1} W_{2}\right) S p W_{3}- \\
-S p\left(W_{3} W_{1}\right) S p W_{2}-S p\left(W_{2} W_{3}\right) S p W_{1}+ \\
\quad+S p\left(W_{1} W_{2} W_{3}\right)+S p\left(W_{3} W_{2} W_{1}\right)
\end{gathered}
$$

where $\left(W_{i}\right)_{\beta}^{\alpha}$ is the ordered exponent along the contour formed by the paths of $i$-th quark and $\Gamma$.1

The averaging over background was done using the cluster expansion method [3], generalized for the case of more than one Wilson loop as well as for a loop with self

\footnotetext{
${ }^{1}$ In what follows $\Gamma$ will be interpreted as the path of the junction.
} 
intersection in [7]. The main result of this method is that under assumption of existence of finite gluonic correlation length $T_{g}$ the generalized area law asymptotics may be obtained. For the Wilson loop (6) we write

$$
<W>_{B}=\int D z_{0} \exp \left(-\sigma S_{1}-\sigma S_{2}-\sigma S_{3}\right)
$$

where $\sigma$ is the string tension and $S_{i}$ is the minimal area inside the contour $C_{i}$, and we integrate in (7) over the junction trajectories $\left\{z_{0}\right\}$ in accordance with our intention to treat a junction as a degree of freedom. The area law (7) is held for the contours with average size much larger than $T_{g}$, and is violated only when the contours are nearly embedded into the same plane.In what follows we neglect such special configurations.

We would like to note here, that the standard approach to the junction motion corresponds to taking the classical trajectory $z_{0}(\tau)$ in the integral $(7)$ or, equivalently, assuming $\sum_{i=1}^{3} S_{i}=\min$. The latter condition can be reduced in the potential model to the assumption that the sum of distances between quarks and junction is minimal. In the "string-type" language the representation (7) means that we are interested in the special kinds of string excitations, which are absent for simple $q \bar{q}$ string configurations, and reveal themselves in multiquark systems.

Using the parametrization

$$
z_{i \mu}=\left(\tau, \vec{r}_{i}\right), \quad z_{0 \mu}=\left(\tau, \vec{r}_{0}\right)
$$

and introducing new dynamical variables

$$
\mu_{i}(\tau)=\frac{T}{2 s_{i}} \dot{z}_{i 0}(\tau) \quad 0 \leq \tau \leq T
$$

we rewrite the Green function (4) as

$$
G=\int D \vec{r}_{1} D \vec{r}_{2} D \vec{r}_{3} D \vec{r}_{0} D \mu_{1} D \mu_{2} D \mu_{3} \exp (-A)
$$

with the effective action

$$
A=\int_{0}^{T} d \tau\left[\sum_{i=1}^{3}\left(\frac{m_{i}^{2}}{2 \mu_{i}}+\frac{\mu_{i} \dot{r}_{i}^{2}}{2}+\frac{\mu_{i}}{2}+\sigma \int_{0}^{1} d \beta_{i} \sqrt{\dot{w}_{i}^{2} w_{i}^{\prime 2}-\left(\dot{w}_{i} w_{i}^{\prime}\right)^{2}}\right)\right]
$$

where the surfaces $S_{i}$ are parametrized by the coordinates $w_{i \mu}$, and $\dot{w}_{i \mu}=\frac{\partial w_{i \mu}}{\partial \tau}, w_{i \mu}^{\prime}=$ $\frac{\partial w_{i \mu}^{\prime}}{\partial \beta}$. This procedure of reduction the four-dimensional dynamics to the three-dimensional one (9) was first suggested in [4] to analyse the $q \bar{q}$ system. With the straight-line ansatz for the minimal surfaces

$$
w_{i \mu}=z_{i \mu}(\tau)\left(1-\beta_{i}\right)+z_{0 \mu}(\tau) \beta_{i}
$$

we obtain the Lagrangian

$$
L=\sum_{i=1}^{3}\left(\frac{m_{i}^{2}}{2 \mu_{i}}+\frac{m_{i} \dot{r}_{i}^{2}}{2}+\frac{\mu_{i}}{2}+\sigma \rho_{i} \int_{0}^{1} d \beta_{i} \sqrt{1+l_{i}^{2}}\right),
$$




$$
\overrightarrow{l_{i}}=\frac{1}{\rho_{i}}\left[\vec{\rho}_{i} \times\left(\left(1-\beta_{i}\right) \dot{\vec{r}}_{i}+\beta_{i} \dot{\vec{r}}_{0}\right)\right], \quad \vec{\rho}_{i}=\vec{r}_{i}-\vec{r}_{0}
$$

The Lagrangian (10) with the additional requirement $\sum_{i=1}^{3} \rho_{i}=\min$ was discussed in detail in [8] with neglecting the angular velocities $\vec{l}_{i}$ under the square roots (string correction), and the attempt to account the string correction was undertaken in [9].

Here we concentrate on the dynamics of junction, so we assume the quarks to be heavy enough $\left(m_{i} \gg \sqrt{\sigma}\right)$ to allow the adiabatic treatment of the problem. Following again the proceduce of [4] we introduce the auxiliary fields $\nu_{i}, \eta_{i}$ :

$$
\begin{aligned}
& G=\int \prod_{i=1}^{3} D \nu_{i} \prod_{i=1}^{3} D \eta_{i} \prod_{i=1}^{3} D \mu_{i} D \vec{r}_{1} D \vec{r}_{2} D \vec{r}_{3} D \vec{r}_{0} \exp (-A[\{\nu\},\{\eta\}]), \\
& A[\{\nu\},\{\eta\}]=\int_{0}^{T} d \tau \sum_{i=1}^{3}\left(\frac{m_{i}^{2}}{2 \mu_{i}}+\frac{\mu_{i} \dot{r}_{i}^{2}}{2}+\frac{\mu_{i}}{2}+\int_{0}^{1} d \beta_{i} \frac{\sigma^{2}\left(\vec{r}_{i}-\vec{r}_{0}\right)^{2}}{2 \nu_{i}}+\right. \\
& \left.+\frac{1}{2} \int_{0}^{1} d \beta_{i} \nu_{i} \eta_{i}^{2}\left(\vec{r}_{i}-\vec{r}_{0}\right)^{2}+\int_{0}^{1} d \beta_{i} \nu_{i} \eta_{i}\left(\vec{r}_{i}-\vec{r}_{0}, \beta_{i} \dot{\vec{r}}_{0}+\left(1-\beta_{i}\right) \dot{\vec{r}}_{i}\right)\right)
\end{aligned}
$$

The integration over the fields $\nu_{i}$ and $\eta_{i}$ actually amounts to taking extremal values in $\nu_{i}$ and $\eta_{i}$.

To make the algebra as simple as possible we consider the case of equal quark masses. Moreover, we take into account only the lowest symmetric hyperspherical harmonic [10] in the quark subsystem, an approximation which is proved to be very successful in few-body systems. It is quite clear that under all these assumptions one has

$$
\mu_{1}=\mu_{2}=\mu_{3}=\mu, \nu_{1}=\nu_{2}=\nu_{3}=\nu, \eta_{1}=\eta_{2}=\eta_{3}=\eta
$$

and, after integrating over the fields $\mu$ and $\eta$ we arrive to the relatively simple Hamiltonian in the center-of-mass frame (in the Minkowski space):

$$
\begin{gathered}
H=3 m+\frac{p^{2}+\frac{3}{4} q^{2}}{2 m}+H_{j} \\
H_{j}=\frac{Q^{2}}{2 m_{s}}+\frac{r^{2}}{\rho^{2}} \frac{(\vec{Q} \vec{n})^{2}}{2 m_{s}}+\frac{1}{2} m_{s} \omega^{2}\left(r^{2}+\rho^{2}\right)+\frac{3}{2} \int_{0}^{1} d \beta \nu, \\
m_{s}=\int_{0}^{1} d \beta \nu \beta^{2}, \quad m_{s} \omega^{2}=\sigma^{2} \int_{0}^{1} \frac{d \beta}{\nu},
\end{gathered}
$$

where the quark wave finction depends only on the hyperradius $\rho, \rho^{2}=\left(\vec{r}_{1}-\vec{r}_{2}\right)^{2}+$ $\left(\vec{r}_{3}-\frac{\vec{r}_{1}+\vec{r}_{2}}{2}\right)^{2}, \quad \vec{r} \equiv \vec{r}_{0}, \vec{n}=\frac{\vec{r}}{r}$, and $\vec{p}, \vec{q}$ and $\vec{Q}$ are the momenta conjugated to the Jacobi coordinates $\vec{r}_{1}-\vec{r}_{2}, \quad \vec{r}_{3}-\frac{\vec{r}_{1}+\vec{r}_{2}}{2}$ and $\vec{r}$ correspondingly.

The adiabatic approximation means that one should find the eigenenergies $E_{n}$ of the "fast" subsystem (13) as functions of $\rho$, and after that substitute $E_{n}(\rho)$ as adiabatic potentials into the Hamiltonian (12) of "slow" subsystem. To perform this program we first found the classical equations of motion for the Hamiltonian (13) with the result ( we restrict ourselves with the spherically symmetric case of $L=0)$ : 


$$
\begin{gathered}
E(a)=\frac{1}{2} m_{s} \omega^{2} \rho^{2}\left(a^{2}+1\right)+\frac{3}{2} \int_{0}^{1} d \beta \nu, \\
(\vec{Q} \vec{n})=m \omega \rho \sqrt{\frac{a^{2} \rho^{2}-r^{2}}{\rho^{2}+r^{2}}}
\end{gathered}
$$

where $a$ is the constant of integration.

It may be shown from equations (14), (15) that "freezing" of the junction degree of freedom actually means substitution the lowest classical energy $E(0)$ into the Hamiltonian (12). In this case integration over $\nu$ is trivial, and one arrives to the potential model Hamiltonian [1][]

$$
H=3 m+\frac{p^{2}+\frac{3}{4} q^{2}}{3 m}+\sqrt{3} \sigma \rho
$$

On the contrary, the Bohr-Zommerfeld quantization procedure for the classical solution (14),(15) yields

$$
\begin{gathered}
E_{n}=\frac{1}{2} m_{s} \omega^{2} \rho^{2}\left(a_{n}^{2}+1\right)+\frac{3}{2} \int_{0}^{1} d \beta \nu, \\
m_{s} \omega \rho^{2} J\left(a_{n}\right)=\left(2 n+\frac{3}{2}\right) \frac{\pi}{2},
\end{gathered}
$$

where

$$
J(a)=\int_{0}^{a} d x \sqrt{\frac{a^{2}-x^{2}}{1+x^{2}}},
$$

and may be expressed in terms of elliptic integrals $\mathbf{K}\left(\frac{a}{\sqrt{1+a^{2}}}\right)$ and $\mathbf{E}\left(\frac{a}{\sqrt{1+a^{2}}}\right)$. The integration over $\nu$ now cannot be performed analytically, apart from the case of asymptotically large $\rho, \rho \gg \frac{1}{\sqrt{\sigma}}$, where the spectrum (17) becomes the spectrum of harmonic oscillator, and the adiabatic potential takes the form

$$
E_{n}(\rho)=\sqrt{3} \sigma \rho+\left(2 n+\frac{3}{2}\right) \cdot \frac{3}{\rho} .
$$

We can find, however, the value of $\rho$ that corresponds to the minimum of the potential well for quarks:

$$
\rho_{0}^{2}=\frac{\pi\left(2 n+\frac{3}{2}\right)}{2 m_{s} \omega^{2} J\left(a^{(0)}\right)}
$$

where for any $\mathrm{n} a^{(0)} \approx 2.2$ is the root of the equation

$$
2 a J(a)-\left(a^{2}+1\right) \frac{\partial J(a)}{\partial a}=0
$$

\footnotetext{
${ }^{2}$ The coefficient at the confinement form in (16) differs slightly from one obtained by taking lowest hyperspherical harmonic of the interaction with $\sigma \sum_{i=1}^{3} \rho_{i}=\min$. It happens because we integrate over the field $\nu$ after hyperspherical decomposition rather than before. The order of these procedures does not matter only if one does not truncate the basis.
} 
Performing the integration over $\nu$ for $\rho_{0}$ defined from the equation $(21)$, we arrive to

$$
\begin{gathered}
\nu_{e x t}=\sqrt{\frac{\sigma}{3}\left(2 n+\frac{3}{2}\right) \frac{a^{(0)^{2}+1}}{J\left(a^{(0)}\right)}}\left(1-\beta^{2}\right)^{-1 / 2}, \\
\rho_{0}^{2}=\frac{4\left(n+\frac{3}{4}\right)}{\sigma J\left(a^{(0)}\right)}, \quad E_{n}=\sqrt{\frac{3 \pi^{2} \sigma}{4}\left(2 n+\frac{3}{2}\right) \frac{a^{(0)^{2}}+1}{J\left(a^{(0)}\right)}}
\end{gathered}
$$

Having in mind that our quarks are heavy $(m \gg \sqrt{\sigma})$ one should expand the adiabatic potential $E_{n}(\rho)(17)$ around $\rho_{0}$ given by (23) using the extremal $\nu_{\text {ext }}$ up to the terms $\sim\left(\rho-\rho_{0}\right)^{2}$. In such a way each adiabatic potential $E_{n}(\rho)$ gives rise to the whole family of excitations in the quark subsystem.

Strictly speaking, the adiabatic potentials (17) are valid either for $n \gg 1$, or for

$\rho \gg \frac{1}{\sqrt{\sigma}}$, where the spectrum (20) is exact. It is known, however, that quasiclassical results are usually rather accurate for small excitation numbers. Using with a lot of cautions the equations $(22)$, (23) for the ground state energy $E_{0}(\rho)$, we arrive to the effective one-dimensional adiabatic potential:

$$
V(\rho)=\frac{\mathcal{L}^{2}}{2 m \rho^{2}}+E_{0}(\rho)
$$

where $\mathcal{L}^{2}=15 / 4$ defines the "hypercentrifugal" barrier for the lowest hyperspherical harmonic [10]. In accordance with equations (17), (18) adiabatic potentials diverge at small $\rho$, or at large $a_{n}^{2}$, but this divergence is only the logarithmic one, as it is easily seen from the asymptotical behaviour of $J(a)$ (see equation (19)). So for "realistic" values of quark masses hypercentrifugal barrier dominates the effective potential (24) at small values of $\rho$. Nevertheless, the spectrum of the ground state family differs substantially from the one given by the potential model (16), and it happens due to the account of junction zero oscillations.

The confining force is not the whole story, and should be supplied by the Couloumb pair-wise interaction at small distances. Moreover, at small $\rho$ the area law (7) is strongly contaminated by the effects of finite correlation length $T_{g}$, so that the string regime does not develop itself at full scale. The explicit calculations which take into account both these effects are in progress now, and will be reported elsewhere. It would be rather interesting to study the $q q q$ system of light quarks in the presented approach, but this problem is much more formidable because the adiabatic approximation is not applicable for the light quarks.

We acknowledge the extremely useful discussions with K.G.Boreskov, O.V.Kancheli and Yu.A.Simonov. This research is supported by the Russian Fundamental Research Foundation, Grant No 93-02-14937, and by INTAS - 93-0079.

\section{References}

[1] J.Carlson, J.Kogut and V.R.Pandharipande, Phys. Rev. D27, 233 (1983) I.Yu.Kobzarev, B.V.Martemyanov, M.G.Shepkin, Yad. Fiz. 40, 1066 (1984) 
[2] N.Isgur, J.Paton, Phys. Rev. D 31, 2910 (1985)

[3] H.G.Dosch and Yu.A.Simonov, Phys. Lett. B205, 339 (1988)

Yu.A.Simonov, Nucl. Phys. B307, 512 (1988), B324, 67 (1989) Yu.A.Simonov, Yad. Fiz. 54, 192 (1991)

[4] A.Yu.Dubin, A.B.Kaidalov and Yu.A.Simonov, Phys. Lett. B323, 41 (1994)

E.L.Gubankova and A.Yu.Dubin, Phys. Lett. B334, 180 (1994)

[5] Yu.S.Kalashnikova, Yu.B.Yufryakov, ITEP-35-95, hep-ph/9506269, Phys. Lett. B in press.

[6] R.P.Feynman, Phys. Rev. 80 , 614 (1950)

J.Schwinger, Phys. Rev. 82, 664 (1951)

Yu.A.Simonov and J.A.Tjon, Ann. Phys. 228, 1 (1993)

[7] A.Yu.Dubin, Yu.S.Kalashnikova, ITEP-40-94, hep-ph/9406332, Yad. Fiz. in press.

[8] Yu.A.Simonov, Phys. Lett. B228, 413 (1989),

M.Fabre de la Riplle, Yu.A.Simonov, Ann. Phys. 212, 235 (1991)

[9] N.Brambilla, P.Consoli, G.M.Prosperi, Phys. Rev. D50, 5878 (1994)

N.Brambilla, G.M.Prosperi, A.Vairo, CERN-TH-95-194, IFUM-511-FT, hepph/9507400

[10] Yu.A.Simonov, Yad. Fiz. 3, 161 (1966), 7, 722 (1968)

A.M.Badalyan, Yu.A.Simonov, Yad. Fiz. 3, 755 (1966)

F.Calogero, Yu.A.Simonov, Phys. Rev. 169, 789 (1968) 